\title{
Análisis del uso de distintos recursos en una web didáctica
}

\section{Analysis of the use of different resources in a didactic website}

\author{
Francisco Cabello Luque ${ }^{1}$, Ana Vanesa Valero García ${ }^{2}$ \\ ${ }^{1}$ Departamento de Psicología Evolutiva y de la Educación, Universidad de Murcia, España (fcabello@um.es) \\ ${ }^{2}$ Departamento de Psicología Evolutiva y de la Educación, Universidad de Murcia, España (vanesavg@um.es)
}

Recibido el 29 de mayo de 2018; revisado el 1 de abril de 2019; aceptado el 8 de abril de 2019; publicado el 1 de diciembre de 2019

\section{RESUMEN:}

A la hora de analizar el uso que los estudiantes hacen de las herramientas docentes online, como es el caso de las webs didácticas, frecuentemente se recurre a las estadísticas globales de acceso. Si bien son medidas adecuadas, resultan poco específicas y no permiten conocer si hay diferencias en función de los tipos de recursos que el alumno tiene disponibles. El objetivo de este trabajo fue comparar el uso que los alumnos hacen de distintos tipos de recursos en una web didáctica, distinguiendo entre recursos evaluados en el examen final, recursos para el trabajo en el aula, recursos de ampliación no evaluados y recursos online de ampliación. Participaron 96 estudiantes (87 mujeres, 9 hombres) matriculados en una asignatura perteneciente al Grado en Logopedia y en la que se utilizó una web didáctica como herramienta para complementar la docencia presencial. Como medidas, se tomaron el número total de accesos a la web didáctica y el promedio de consultas a cada tipo de tipo de recursos. Los resultados mostraron que, si bien a nivel global hubo un uso intenso, frecuente y estable de la web, al mismo tiempo hubo algunos recursos que apenas fueron consultados por los alumnos. Por tanto, se sugiere que la disponibilidad de un recurso de aprendizaje determinado en una web didáctica no supone que vaya a ser utilizado y que la forma de aumentar el uso de un recurso es evaluándolo o utilizándolo directamente en el aula.

PALABRAS CLAVE: SITIOS WEB, WEBS DIDÁCTICAS, BLENDED LEARNING, RECURSOS DE APRENDIZAJE. ABSTRACT:
To analyze how students use online learning tools, such as didactic websites, a common practice is to report global access statistics. Although these are adequate measures, they are not specific enough and do not allow to determine if there are differences depending on the type of resources available to students. The aim of this study is to compare how students use different types of resources in a didactic website, distinguishing between resources to be evaluated in a final exam, resources for work in the classroom, extension resources not to be evaluated, and online extension resources. To this end, the 96 students (87 women, 9 men) taking part in the research enrolled in a subject that is part of the Degree in Speech Therapy, and where a didactic website was used as a tool to complement face-toface teaching. The total number of accesses to the didactic website and the average number of reads of each type of resources were taken as units of measure. The results indicated that, although the number of visits generally reflected an intense, frequent, stable use of the website, at the same time there were some types of resources that were hardly used by students. Thus, it is suggested that the availability of a particular learning resource on a didactic website does not imply that it will be used, and that the best way to increase the use of a resource is to evaluate it, or to use it in the classroom.

KEYWORDS: WEB SITES, DIDACTICAL WEBSITES, BLENDED LEARNING, LEARNING RESOURCES. 


\section{INTRODUCCIÓN}

La innovación a través de las TIC es una de las prácticas que, con mayor frecuencia, se ha aplicado a la hora de buscar la mejora de la docencia universitaria (Enguita y Cruz, 2005; García-Sanz, García-Sánchez, Maquilón y Morillas; Marín y Reche, 2011; Oliver, 2001; Oliver y Herrington, 2003; Reinoso, 2009) y, en este sentido, son numerosas las herramientas basadas en las nuevas tecnologías que se han aplicado en el aula (Area, Castro, De la Cruz, Sanabria y Estévez, 2003; Ríos, Castañeda y Roca, 2003).

En este contexto de uso de las TIC encontramos las webs didácticas, que se pueden conceptualizar como páginas web que contienen toda la información acerca del desarrollo y evaluación de una asignatura, que permiten facilitar la enseñanza y el aprendizaje y que sirven de guía al alumno (Bueno y Gil, 2007; García-Sánchez, MartínezJuárez y Martínez-Segura, 2008; García-Sánchez y Martínez-Segura, 2009; Marqués, 2005; Mur y Serrano, 2006).

Por tanto, esta herramienta emplea una página web como elemento fundamental y trata de aprovecharse de sus posibilidades mediante la inclusión de imágenes, videos o enlaces; sin embargo, no se trata de un mero listado de archivos para descargar con los contenidos de la asignatura, sino que incluye toda una serie de aspectos como competencias y contenidos a adquirir, itinerarios de aprendizaje a seguir, los recursos completos, o la secuencia de actividades de aprendizaje propuesta. En este sentido, está pensada para apoyar y fortalecer las experiencias de formación presenciales, de acuerdo con los planteamientos del blended learning (Garrison y Vaughan, 2008; Horn y Staker, 2017; Martín, 2014; Salinas, de Benito, Pérez, y Gisbert, 2018; Snart, 2010). E incluso, estaría bordeando conceptos más novedosos como el de los entornos personales de aprendizaje (Castañeda y Adell, 2013; Castañeda, Dabbagh y Torres-Kompen, 2017; Ruiz-Palmero, Sánchez y Gómez, 2013).

Son numerosas las investigaciones que informan de su aplicación en docencia universitaria y de sus efectos positivos en el proceso de enseñanzaaprendizaje. Cabello, García-Sánchez y Peñalver (2018) recogen una síntesis de las principales ventajas del uso de las webs didácticas: (1) la frecuencia de su uso; (2) la visión positiva que tienen de las mismas tanto alumnos como docentes; (3) su reconocimiento por parte de estos dos grupos como una herramienta útil; (4) la existencia de una relación positiva entre una mayor implicación en el uso de la web didáctica y una mejor calificación en la asignatura; (5) el hallazgo de una una mayor tasa de presentación a los exámenes de las asignaturas que las emplean y (6) la facilitación del uso de metodologías activas y participativas en el aula (Area, 2001; Area, Castro, De la Cruz, Sanabria, y Estévez, 2003; Cabello, García Sánchez, y Mirete, 2009; García-Sánchez, Mirete, y Maquilón, 2013; Maquilón, Mirete, García-Sánchez y Hernández, 2013; Mirete y García-Sánchez, 2014; Mirete, Cabello, y García-Sánchez, 2009; Mirete, GarcíaSánchez, y Maquilón, 2014; Mirete, García-Sánchez, y Sánchez-López, 2011; Moreno, Martínez y Martín, 2004; Moreno, Martínez, Martín y Trigo, 2005; Pérez, 2000; Río, Sanz y Búcari, 2017; Ríos, Castañeda y Roca, 2003; Terceiro, 1996; Rodríguez, 2017).

Como se acaba de mencionar, un hallazgo común es que las webs didácticas son empleadas de forma frecuente y constante por el alumnado, generalmente de forma semanal y a lo largo de todo el cuatrimestre. Sin embargo, como medida de ese uso se ofrecen habitualmente datos globales como son el número de visitas a la web o sus herramientas, sin que exista un análisis más detallado. Dado que una de las características más destacables de las webs didácticas es la posibilidad de ofrecer diferentes recursos apoyándose en las nuevas tecnologías (por ejemplo, artículos científicos, lecturas de ampliación y complementarias, videos didácticos o enlaces a páginas web con información), una medida basada simplemente en el número total de visitas no permite determinar si realmente se están utilizando todos estos distintos recursos o si algunos se emplean más que otros.

Por tanto, en este trabajo se analiza el uso diferencial de varios tipos de recursos ofrecidos en una web didáctica diseñada para una asignatura universitaria. Para ello se ofrecen las habituales estadísticas de acceso total pero, al mismo tiempo, se lleva a cabo un análisis específico de si los alumnos utilizan los diferentes recursos ofrecidos en la web, en función de si forman parte de los contenidos evaluados en el examen, de su obligatoriedad, de si son trabajados en clase o no, o de su carácter de ampliación.

\section{MÉTODO}

\subsection{Participantes}

En este estudio participaron 96 estudiantes, 87 mujeres (edad media $=21.30$ años, DT $=4.16$ años $)$ y 9 hombres $($ edad media $=21.78$ años, $\mathrm{DT}=2.58$ 
años). No hubo diferencias significativas en la edad entre hombres y mujeres, $\mathrm{t}(94)=-0.338, \mathrm{p}=0.495$.

Todos los alumnos estaban matriculados por primera vez en la asignatura "Alteraciones logopédicas en trastornos del desarrollo" del Grado en Logopedia de la Universidad de Murcia. Esta asignatura se imparte durante el segundo cuatrimestre del primer curso, tiene carácter obligatorio y supone una carga de 6 ECTS.

\subsection{Instrumentos}

\subsubsection{Web didáctica}

La web didáctica empleada era accesible a través del Aula Virtual de la Universidad de Murcia y ofrecía las siguientes herramientas:

- Información imprescindible de la asignatura, como las competencias, los objetivos, la metodología que se va a seguir o los procedimientos de evaluación.

- Herramientas de comunicación: incluían tanto un tablón de noticias como herramientas de mensajería para permitir el adecuado contacto con el docente y con el resto de los compañeros.

- Cronograma: consistía en una descripción, día por día, de las actividades que se iban a desarrollar en clase tanto en la semana actual como en la siguiente.

- Materiales: el contenido completo de la asignatura estuvo disponible desde el comienzo del cuatrimestre, incluyendo tanto documentos (evaluables y de ampliación) como materiales de apoyo, imágenes o videos.

- Prácticas: donde se ofrecían los materiales necesarios para la realización de las prácticas de cada asignatura, así como información sobre cómo realizarlos y las fechas de entrega.

- Enlaces de ampliación: permitían el acceso a información adicional sobre la asignatura en forma de lecturas o enlaces a webs externas.

Esta estructura sigue las recomendaciones de numerosos autores sobre los elementos que deberían de configurar este tipo de webs (Bueno y Gil, 2007; Cabero, Llorente y Román, 2004; García-Sánchez y Martínez-Segura, 2009; García-Sánchez, MartínezJuárez y Martínez-Segura, 2008; Marcelo, Puente, Ballesteros y Palazón, 2003; Marqués, 2005; Martínez Segura, García Sánchez y Martínez Juárez, 2008; Román, 2000).

Las figuras 1, 2 y 3 ofrecen varias capturas para mostrar el aspecto de la web que los alumnos utilizaron.

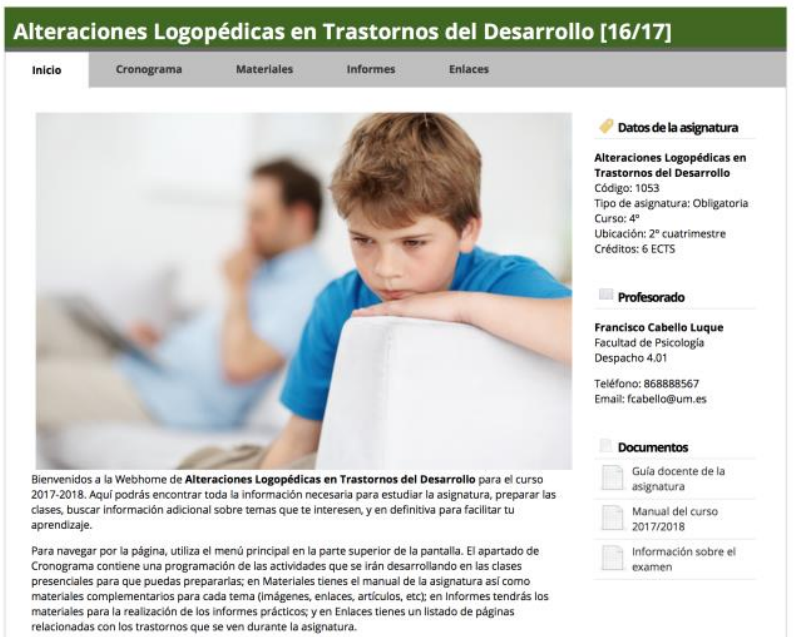

Figura 1. Página de bienvenida de la web didáctica

Z Semana del 27/Febrero al 5/Marzo
\begin{tabular}{|l|l|l|}
\hline Día & ¿Qué se vio en clase? & \multicolumn{2}{|c|}{ ¿Qué actividades se hicieron? } \\
\hline Miércoles 1 & $\begin{array}{l}\text { Análisis de casos de TEL y retraso } \\
\text { en el lenguaje }\end{array}$ & $\begin{array}{l}\text { Actividades 13 a 15 (Casos de TEL } \\
\text { y retraso del lenguaje) }\end{array}$ \\
\hline Jueves 2 & $\begin{array}{l}\text { Último caso de TEL y retraso del } \\
\text { lenguaje. Comienzo del Tema 3. } \\
\text { Exposición sobre problemas de } \\
\text { lenguaje asociados a } \\
\text { multiculturalidad y en adopción } \\
\text { internacional }\end{array}$ & $\begin{array}{l}\text { Actividades 16 y 17 (Casos de TEL } \\
\text { y retraso del lenguaje) y Actividad } \\
18 \text { (Aulas de acogida de la Región } \\
\text { de Murcia) }\end{array}$ \\
\hline
\end{tabular}

SEMINARIO 3: Análisis de casos de TEL y retraso en el lenguaje

Figura 2. Ejemplo de semana en el cronograma

\section{Tema 2 - TEL y retrasos del lenguaje}

Tema 2 completo

\section{Materiales del tema}

- Clasificación de tipos de TEL

- Resumen de criterios para el diagnóstico

Lecturas obligatorias

PDiversidad dentro del TEL

\section{Lecturas de ampliación}

- Subtipos del TEL en hispanohablantes

- Guía para la evaluación del TEL

Enlaces

ASHA - Spoken language disorders

\section{Videos sobre TEL}

- Introducción a la campaña RALLI

- No es tu culpa - Español

Asociaciones de atención a niños con TEL AMUTELHA (Murcia)

AVATEL (Valencia)

Figura 3. Materiales ofrecidos para uno de los temas 


\subsubsection{Tipos de recursos}

Los diferentes recursos ofrecidos en la web didáctica se dividieron en cuatro grupos, en función de si eran evaluados en el examen, se trabajaban en clase, tenían carácter de ampliación, o eran recursos multimedia y enlaces web también para ampliación:

- Tipo 1. Recursos que eran evaluados en el examen final (apuntes de cada tema y lecturas obligatorias).

- Tipo 2. Recursos no evaluados en el examen, pero empleados en actividades de clase (fundamentalmente material para análisis de casos).

- Tipo 3. Recursos de ampliación no evaluados y que tampoco se trabajaban en clase (en forma de lecturas y artículos científicos).

- Tipo 4. Recursos multimedia (videos e imágenes) y enlaces a páginas externas.

La tabla 1 muestra el número de recursos, dentro de cada uno de esos tipos, que estaban incluidos en la web. Dado que la asignatura constaba de un total de 6 temas, puede verse que hay uno o dos recursos de Tipo 1, Tipo 2 y Tipo 3 por cada tema, y un número algo más elevado de Tipo 4.

Tabla 1. Número de recursos para cada tipo

\begin{tabular}{cc}
\hline Tipo & Núm. recursos \\
\hline Tipo 1 & 10 \\
Tipo 2 & 5 \\
Tipo 3 & 12 \\
Tipo 4 & 36 \\
\hline
\end{tabular}

\subsection{Procedimiento}

La web didáctica fue aplicada empleando el procedimiento descrito por Cabello, García-Sánchez y Peñalver (2018) de manera que su utilización formaba parte de la propia dinámica de la asignatura:

1. Al comenzar cada semana lectiva, se sugería al alumno que entrara en la web y consultara el cronograma para saber qué se iba a trabajar y qué partes del temario debía consultar.

2. Una vez que conocía lo que se iba a hacer, el alumno debía entrar en la sección de contenidos para revisar los materiales y consultarlos antes de la clase presencial.

3. En las clases se permitía tener los recursos de aprendizaje para ir haciendo alguna anotación a los mismos (incluso con un portátil o una tableta), aunque el trabajo del aula se basaba en metodologías más activas y participativas.
4. Por último, y una vez terminadas las clases, se actualizaba el cronograma para reflejar lo que se había trabajado y lo que se iba a hacer en las siguientes semanas.

El análisis del uso que los estudiantes hicieron de los diferentes recursos se basó en estadísticas que eran recopiladas automáticamente por el Aula Virtual de la Universidad de Murcia y que se obtuvieron al finalizar cada cuatrimestre.

\section{RESULTADOS}

\subsubsection{Uso total de la web didáctica}

En primer lugar, se ofrecen los resultados globales de acceso a la web didáctica, siendo el tipo de estadística más habitual con la que se estudia el uso de los recursos online.

En total, los estudiantes accedieron a la web didáctica 3940 veces. Para representar esos accesos a lo largo del cuatrimestre, se calculó el promedio de accesos por cada alumno durante cada una de las 14 semanas en las que se impartió docencia, tal y como aparece en la figura 4.

Analizando esta representación de los datos de accesos encontramos (1) que los alumnos accedieron a la web didáctica un número muy elevado de ocasiones, (2) que todas las semanas hubo, de promedio, al menos dos accesos por cada alumno matriculado y (3) que el número de accesos semanales se mantuvo constante durante todo el cuatrimestre, con incrementos en las semanas 7 y 12 debido a la entrega de trabajos. Por tanto, los datos totales sugieren claramente un uso habitual de la web didáctica como herramienta para complementar las clases presenciales.

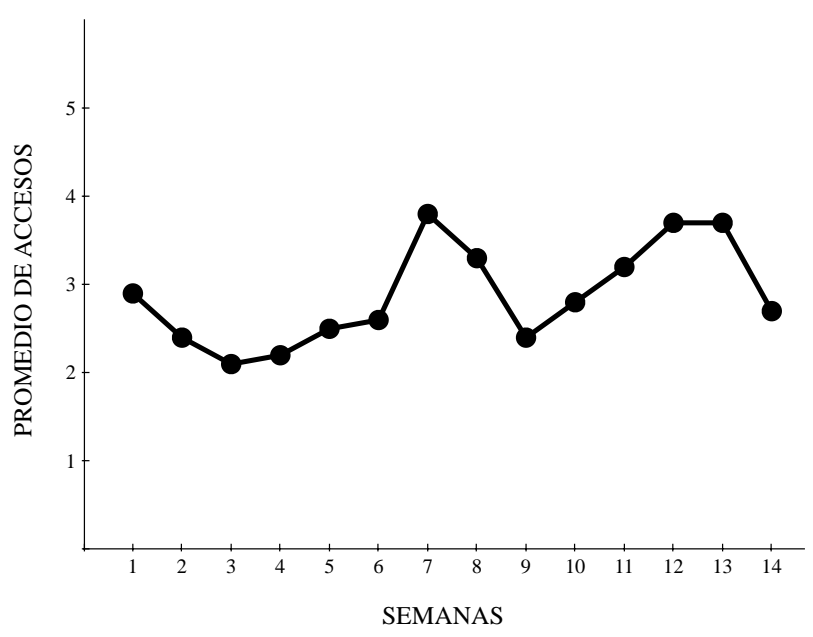

Figura 4. Promedio de accesos por alumno durante el cuatrimestre (14 semanas) 


\subsubsection{Uso por tipo de recursos}

En segundo lugar, se llevó a cabo un análisis más detallado de los accesos, centrado en estudiar las veces que los estudiantes habían consultado los distintos tipos de recursos.

Dado que dentro de cada tipo el número de recursos era distinto, se calculó el promedio de consultas a cada recurso por cada alumno matriculado.

En la figura 5 aparece representado ese promedio, de manera que puede verse cuántas veces se consultó cada uno de los recursos ofrecidos en cada tipo.

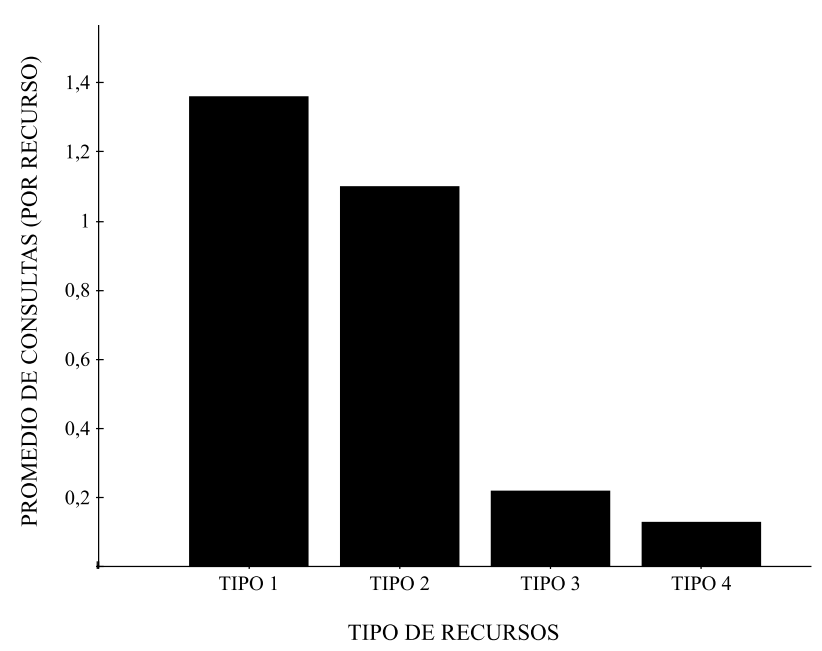

Figura 5. Promedio de consultas por alumno a cada tipo de recursos de aprendizaje

A partir del análisis visual de los datos se aprecia que los recursos Tipo 1 (aquellos evaluados en el examen final) y Tipo 2 (los empleados para las actividades de clase) son consultados al menos una vez por todos los alumnos; mientras, los recursos Tipo 3 (lecturas de ampliación no evaluadas) y Tipo 4 (videos, imágenes y enlaces a páginas web) fueron mucho menos consultados, siendo estos últimos los que obtuvieron menor promedio de consultas.

Se llevó a cabo un ANOVA de medidas repetidas, en el que la prueba de Mauchly mostró que no se cumplió el supuesto de esfericidad para el efecto principal de tipo de recurso $\left(X^{2}(2)=49.36, p\right.$ $<.001) \mathrm{y}$, por tanto, los valores de significación estadística deben ser corregidos.

El análisis de varianza mostró entonces un efecto significativo del tipo de recurso $(\mathrm{F}(2.35,223.41)=$ $514.44, \mathrm{p}<.001)$. Se realizaron entonces las comparaciones por pares pertinentes, obteniéndose diferencias estadísticamente significativas en todos los casos, tal y como aparece en la tabla 2.
Tabla 2. Comparaciones por pares entre los distintos tipos de recursos de aprendizaje

\begin{tabular}{ccc}
\hline Comparación & dif & $\mathrm{p}$ \\
\hline Tipo 1-Tipo 2 & 0.265 & $<.001$ \\
Tipo 1-Tipo 3 & 1.146 & $<.001$ \\
Tipo 1-Tipo 4 & 1.230 & $<.001$ \\
\hline Tipo 2-Tipo 3 & 0.882 & $<.001$ \\
Tipo 2-Tipo 4 & 0.965 & $<.001$ \\
\hline Tipo 3-Tipo 4 & 0.084 & $<.01$ \\
\hline
\end{tabular}

Los recursos Tipo 1 fueron significativamente más utilizados que los restantes tipos. Por su parte, los Tipo 2 también fueron más consultados que los Tipo 3 y Tipo 4. En cuanto a los Tipo 3, los alumnos también los usaron significativamente más que los Tipo 4 , que fueron los que presentaron menor frecuencia de uso.

Tomando todos estos datos en consideración, queda claro que los recursos Tipo 1 fueron los más consultados, seguidos a continuación por los Tipo 2 . En cuanto a los recursos Tipo 3 y Tipo 4, apenas fueron consultados por los alumnos, aunque los primeros recibieron más accesos que los segundos.

\section{DISCUSIÓN}

El objetivo inicial del estudio era analizar el uso diferencial que los estudiantes hacían de los distintos tipos de recursos de aprendizaje ofrecidos en una web didáctica. A partir de los resultados descritos más arriba, se desprenden las siguientes conclusiones.

La primera es que la web didáctica es un recurso muy empleado por los estudiantes. El volumen de visitas y (especialmente) la frecuencia y estabilidad de estas demuestran un uso constante a lo largo de todo el cuatrimestre, en el que la mayor parte de las semanas hay al menos dos accesos por alumno de promedio. Es decir, los resultados sugieren que la web didáctica no se está empleando como una mera plataforma para descargar documentos en momentos puntuales, sino como una herramienta de acompañamiento al aprendizaje. Esta frecuencia en el uso, por otra parte, está en consonancia con lo descrito por otros investigadores (Area, Castro, De la Cruz, Sanabria y Estévez, 2003; Cabello, García Sánchez y Mirete, 2009; García-Sánchez, Mirete y Maquilón, 2013; Mirete, García-Sánchez y Maquilón, 2014; Moreno, Martínez y Martín, 2004; Moreno, Martínez, Martín y Trigo, 2005; Ríos, Castañeda y Roca, 2003).

La segunda conclusión es que, tal y como dejan claro los datos presentados, el uso que hacen los alumnos de la web didáctica no es uniforme, sino 
que depende del tipo de recursos. Así, se ha demostrado: (1) que aquellos recursos de aprendizaje que eran evaluados en el examen final de la asignatura, tales como los apuntes de cada tema y las lecturas obligatorias, resultaron los más utilizados por los estudiantes; (2) que los recursos utilizados en el aula también fueron ampliamente consultados y (3) que los recursos que no se evaluaban o usaban en el aula y que simplemente tenían como objetivo la ampliación de conocimientos, apenas eran consultados. Este es un tipo de análisis más detallado que no se ha realizado previamente al estudiar el impacto de una web didáctica y que desde nuestro punto de vista ofrece una mejor perspectiva de cómo los estudiantes usan estas herramientas online.

Por tanto, en cuanto a las características de los recursos que determinan una mayor utilización, el que aparece como más relevante es la evaluación del mismo mediante un examen ( $\mathrm{u}$ otra modalidad similar). La utilización de este tipo de evaluaciones en la docencia universitaria es objeto de un intenso debate (Brown y Glasner, 2003; De Luxán, 1998; Molero, 2004; Pidone, 2017) pero los datos recopilados señalan que la práctica totalidad de los alumnos revisaron los recursos que formaban parte del examen de la asignatura. El trabajo en clase también está relacionado con un mayor uso del recurso por parte de los alumnos, lo que supone un apoyo a la efectividad de metodologías activas en el aula (Capa, Icarán y Bonson, 2005; García y otros, 2012; González, 2013).

Al mismo tiempo, queda claro que los recursos de ampliación y especialmente los videos y enlaces a páginas externos no son apenas consultados. Es un hallazgo relevante porque, con frecuencia, la inclusión de estos elementos se cita como una de las ventajas de las webs didácticas al aprovechar sus características como herramienta online (Area, 2001; Bueno y Gil, 2007; González y Cabrera, 2010; Mirete, García-Sánchez y Maquilón, 2014). Sin embargo, este trabajo arroja dudas sobre la efectividad real de las mismos; de hecho, buena parte de los alumnos simplemente no los consulta en ningún momento. En cualquier caso, se hace necesaria más investigación en este punto para tratar de determinar aspectos como, por ejemplo, qué tipo de alumnos son los que acceden a estos recursos o cómo aumentar su utilización.

Finalmente, otra conclusión que se extrae de los resultados tiene que ver con la adecuación de las estadísticas de acceso como índice del uso de una web didáctica (y, por extensión, de otra herramienta online similar). Si en este trabajo solo se hubieran tenido en cuenta los datos de accesos globales de la muestra de estudiantes, no se habría apreciado el hallazgo de que el uso que hacen no es uniforme, sino que varía significativamente según el tipo de recurso de aprendizaje. Por tanto, un análisis más detallado ha permitido sacar a la luz una riqueza que de otra forma se habría perdido. Las implicaciones de ofrecer sólo datos globales del total de alumnos y la necesidad de contemplar otras medidas para valorar el impacto de una web didáctica han sido expuestas previamente (Cabello, García-Sánchez y Peñalver, 2018; Moreno, Martínez, Martín y Trigo, 2005) pero continúan siendo aspectos sobre los que es necesario seguir trabajando.

Por supuesto, para valorar adecuadamente todos estos hallazgos es necesario tomar en consideración las limitaciones que pudieran afectar a su validez. En este sentido, podemos destacar dos. Primero, el hecho de que la asignatura forma parte de un título de Ciencias de la Salud y tiene un carácter eminentemente práctico, por lo que los resultados pueden no ser aplicables a otras asignaturas que cuenten con condiciones diferentes. Y segundo, las propias características del alumnado, con una amplia mayoría de mujeres, también puede plantear interrogantes respecto a su generalización a otros grupos en los que, por ejemplo, la proporción de hombres sea más elevada. Son limitaciones que, no obstante, pueden aclararse más adelante con futuras investigaciones.

Tal como señalaron Hannan y Silver (1999, 2005), la utilización por parte del docente de un recurso didáctico concreto no garantiza ni su utilización ni tampoco la innovación en el proceso de enseñanza-aprendizaje. Una demostración empírica de esta idea la tenemos en la presente investigación, donde queda claro que no todos los recursos que ofrece una web didáctica son empleados por los alumnos. Muy al contrario, la forma de garantizar que un alumno acceda a y consulte un recurso, es que forme parte del propio funcionamiento y evaluación de la asignatura.

Por tanto, a la luz de los resultados encontrados, aquellos docentes que aplican webs didácticas en docencia universitaria (y probablemente herramientas web en general) no pueden confiar simplemente en que los alumnos vayan a utilizar todos los recursos de aprendizaje disponibles, sino que es necesario favorecer su uso de forma explícita, por ejemplo, mediante la inclusión en la evaluación de la asignatura o mediante su utilización en el aula. 


\section{REFERENCIAS}

Area, M. (2001). Las redes de ordenadores en la enseñanza universitaria. Hacia los campus virtuales. En A. GarcíaValcárcel (Coord.), Didáctica Universitaria (pp. 231260). Madrid: La Muralla.

Area, J., Castro, F. De la Cruz, G., Sanabria, A., y Estévez, RC. (2003). Diseño y experimentación pedagógica de materiales didácticos distribuidos a través de la www. La web docente de la signatura de tecnología educativa. En Actas de la I Jornadas Canarias sobre las TIC en la docencia universitaria. La Laguna: Universidad de La Laguna.

Brown, S. A., y Glasner, A. (Eds.). (2003). Evaluar en la universidad: problemas y nuevos enfoques. Madrid: Narcea Ediciones.

Bueno, C. y Gil, J.J. (2007). Web docente: estructura y procedimientos básicos de gestión eficaz. Revista Interuniversitaria de Formación del Profesorado, 21(1), 37-50.

Cabello, F., García-Sánchez, F.A. y Mirete, A.B. (2009). Valoración de tres experiencias de uso de webs didácticas como herramienta para facilitar la docencia universitaria. En La innovación educativa en el contexto actual de la educación superior (pp. 661-666).. Vigo: Universidad de Vigo.

Cabello, F., García-Sánchez, F.A. y Peñalver, M.D. (2018). Webs didácticas en docencia universitaria: Lecciones tras 10 años de utilización. En Actas del III Congreso Internacional de Innovación Docente. Murcia: Servicio de Publicaciones de la Universidad de Murcia.

Cabero, J., Llorente, M.D.C., y Román, P. (2004). Las herramientas de comunicación en el "aprendizaje mezclado". Pixel-Bit. Revista de Medios y Educación, $23,27-41$.

Capa, A.B., Icarán, E., y Bonson, M. (2005). Metodologías activas. En A. Cruz y A. Benito (Eds). Nuevas claves para la docencia universitaria en el Espacio Europeo de Educación Superior. Madrid: Narcea.

Castañeda, L. y Adell, J. (2013). Entornos Personales de Aprendizaje: claves para el ecosistema educativo en red. Alcoy: Marfil.

Castañeda, L., Dabbagh, N., y Torres-Kompen, R. (2017). Entornos Personales de Aprendizaje: prácticas basadas en la investigación, marcos y desafíos. Journal of New Approaches in Educational Research, 6(1), 1-2.

De Luxán, J.M. (1998). La evaluación de la Universidad de España. Revista de Educación, 315, 11-28.

Enguita, C. y Cruz, A. (2005). Recursos tecnológicos. En A. Cruz y A. Benito (Eds). Nuevas claves para la docencia universitaria en el Espacio Europeo de Educación Superior (pp. 101-124). Madrid: Narcea.

García, J.N., Marbán, J.M., de Caso, A.M., Pacheco, D.I., Robledo, P., Álvarez, L., ... y García-Martín, E. (2011). Innovación en el EEES con metodologías activas. International Journal of Developmental and Educational Psychology, 3(1), 365-372.

García-Sánchez, F.A., Martínez-Juárez, M. y Martínez-Segura, M.J. (2008). Concepto de WEB-home para asignaturas universitarias. En Arnaiz, P., García Sanz, M.P. y Hernández Abenza, L. (Coords.), III Jornadas sobre el Espacio Europeo de Educación Superior: "Avanzando hacia Bolonia”. Murcia: Servicio de Publicaciones de la Universidad de Murcia.
García-Sánchez, F.A. y Martínez-Segura, M.J. (2009). Webdocente y aprendizaje: una experiencia en el contexto de la convergencia al EEES. En R. Roig Vila (Dir.). Investigar desde un contexto educativo innovador (pp. 201.-218). Alcoy: Marfíl.

García-Sánchez, F.A., Mirete, A.B. y Maquilón, J. (2013). Implementación y evaluación de webs didácticas para la docencia universitaria presencial. Revista Electrónica Interuniversitaria de Formación del Profesorado, 16(1), 121-132.

García-Sanz, M.P., García-Sánchez, F.A., Maquilón, J., y Morillas, L. (2010). Diseño de Webs didácticas para la mejora de la calidad de los procesos educativos en el EEES. En J.R. Hilera, F. Cervantes y L. Bengochea (Eds.), CAFVIR 2010 (pp. 191-199).. Alcalá de Henares: Servicio de Publicaciones de la Universidad de Alcalá de Henares

Garrison, D.R. y Vaughan, N.D. (2008). Blended learning in higher education. Framework, principles and guidelines. San Francisco: Jossey-Bass.

González, J.J. (2013). Metodologías activas en la Universidad. Murcia: Servicio de Publicaciones UCAM.

Hannan, A. y Silver, H. (1999). Innovating in Higher Education. Teaching, Learning and Institutional Cultures. Buckingham, UK: Open University Press.

Hannan, A. y Silver, H. (2005) La innovación en la enseñanza superior. Enseñanza aprendizaje y culturas institucionales. Madrid: Narcea.

Horn, M.B., y Staker, H. (2017). Blended: Using disruptive innovation to improve schools. London: John Wiley y Sons.

Marcelo, C., Puente, D., y Ballesteros, M.A. (2003). E-learning teleformación: diseño, desarrollo y evaluación de la formación a través de Internet. Madrid: Gestión 2000.

Marín, V. y Reche, E. (2011). La alfabetización digital del alumnado que accede a la Universidad de Córdoba. Edutec-e. Revista Electrónica de Tecnología Educativa, $35,1-13$.

Marqués, P. (2005). Las webs docentes. Recuperado de: http://dewey.uab.es/pmarques/webdocente.htm

García, A. (Ed.). (2014). Blended learning en educación superior: Perspectivas de innovación y cambio. Madrid: Síntesis.

García-Sánchez, F.A., Martínez-Segura, M.J., y MartínezJuárez, M. (2008). Una WEB-Home como herramienta de enseñanza/aprendizaje: una experiencia en el contexto de la convergencia al EEES. Comunicación presentada a las VI Jornadas de Redes de Investigación en Docencia Universitaria. Alicante, 9 y 10 de Junio.

Maquilón, J., Mirete, A., García-Sánchez, F.A., y Hernández, F. (2013). Valoración de las TIC por los estudiantes universitarios y su relación con los enfoques de aprendizaje. Revista de Investigación Educativa, 31(2), 537-554.

Mirete, A.B. y García-Sánchez, F.A. (2014). Rendimiento académico y TICs. Una experiencia con Webs Didácticas en la Universidad de Murcia. Pixel-Bit. Revista de Medios y Educación, 44, 169-183.

Mirete, A.B., Cabello, F., Martínez-Segura, M.J., y GarcíaSánchez, F.A. (2009). Resultados de la aplicación piloto de un instrumento para la evaluación de webs didácticas en asignaturas universitarias. EDITUM: Universidad de Murcia.

Mirete, A.B., García-Sánchez, A., y Maquilón, J. (2014). Webs didácticas en educación superior: análisis de su contenido y valoración del estudiante. Revista 
Interuniversitaria de Formación del Profesorado, 28(1), 95-114.

Mirete, A.B., García-Sánchez, F.A. y Sánchez-López, C. (2011). Implicación del alumnado en la valoración de su satisfacción con las webs didácticas. Edutec-e. Revista Electrónica de Tecnología Educativa, 37, 1-13.

Molero, D. (2004). La evaluación de la docencia en la universidad. Jaén: Universidad de Jaén.

Moreno, R., Martínez, R., y Martín, I. (2004). Visitas a las páginas de una web docente universitaria. Pixel-Bit. Revista de Medios y Educación, 22, 6.

Moreno, R., Martínez, R., Martín, I, y Trigo, M.E, (2005). Utilidad de una web docente en una materia universitaria presencial. En Libro de Actas del II Congreso Hispano-Portugués de Psicología. Santiago de Compostela: Universidad de Santiago.

Mur, F. y Serrano, C. (2006). Elaboración de una web docente. Recuperado de: http://www.5campus.org/leccion/ webdocente

Oliver, R. (2001). Developing e-learning environments that support knowledge construction in higher education. En $\mathrm{S}$. Stoney y J. Burn (Eds). Working for excellence in the e-conomy. Chuchlands: We-B Centre.

Oliver, R. y Herrington, J. (2003). Exploring technologymediated learning from a pedagogical perspective. Journal of interactive Learning Environments, 11(2), 111-126.

Pérez, J. M. (Comp.) (2000). Comunicación y educación en la sociedad de la información. Madrid: Paidós.

Pidone, C. L. (2017). Evaluación de los aprendizajes en la universidad. Revista diálogos educativos, 5(9), 38-42.

Reinoso, A. J. (2009) Análisis de la incorporación de una plataforma Wiki a la docencia de la asignatura Nuevas Tecnologías de la Información. Red U - Revista de Docencia Universitaria, 5, 86-106

Río, L. D., Sanz, C., y Búcari, N. (2017). Actitudes de los estudiantes frente a un material hipermedial para el aprendizaje de la matemática: un estudio de caso. Revista Iberoamericana de Tecnología en Educación y Educación en Tecnología, 19, 24-33.

Ríos, M.A., Castañeda, R.J., y Roca, J.C. (2003). Apoyo de una web a la docencia presencial: una experiencia en TMRA2. Revista de Enseñanza Universitaria, (22), 4568.

Rodríguez, M.C. (2017). Modelo de enseñanza y aprendizaje de la comunicación profesional universitaria desde la web didáctica. Revista Boletín Redipe, 6(8), 102-114.

Román, P. (2000). Usos de la World Wide Web con fines educativos. Pixel-Bit. Revista de Medios y Educación, 15,5 .

Ruiz-Palmero, J., Sánchez, J., y Gómez, M. (2013). Entornos personales de aprendizaje: estado de la situación en la Facultad de Ciencias de la Educación de la Universidad de Málaga. Píxel-Bit. Revista de Medios y Educación, 42, 171-181.

Salinas, J., de Benito, B., Pérez, A., y Gisbert, M. (2018). Blended learning, más allá de la clase presencial. Revista Iberoamericana de Educación a Distancia, 21(1), 195-213.

Snart, J.A. (2010). Hybrid Learning. The perils and promise of blending online and face-to-face instruction in higher education. California: ABC CLIO.

Terceiro, J.B. (1996).Socied@ddigit@l.Del homo sapiensal homo digitalis. Madrid: Alianza Editorial. 